\title{
Transient functional blood flow change in the human brain measured noninvasively by diffusing-wave spectroscopy
}

\author{
J. Li, ${ }^{1}$ M. Ninck, ${ }^{1}$ L. Koban, ${ }^{2}$ T. Elbert, ${ }^{2}$ I. Kissler, ${ }^{2}$ and T. Gisler ${ }^{1, *}$ \\ ${ }^{1}$ Fachbereich Physik, Universität Konstanz, 78457 Konstanz, Germany \\ ${ }^{2}$ Fachbereich Psychologie, Universität Konstanz, 78457 Konstanz, Germany \\ "Corresponding author: thomas.gisler@uni-konstanz.de
}

\begin{abstract}
Multispeckle diffusing-wave spectroscopy (DWS) is used to measure blood flow transients in the human visual cortex following stimulation by $7.5 \mathrm{~Hz}$ full-field and checkerboard flickering. The average decay time $\tau_{\mathrm{d}}$ characterizing the decay of the DWS autocorrelation function shows a biphasic behavior; within about $2 \mathrm{~s}$ after stimulation onset, $\tau_{\mathrm{d}}$ increases rapidly to about $6 \%$ above the baseline value. At later times, $\tau_{\mathrm{d}}$ slowly decreases and reaches a steady-state value about $5 \%$ below the baseline value after about $15 \mathrm{~s}$. The initial increase of the DWS signal suggests a transient reduction of the cortical blood flow velocity shortly after stimulation onset. Measurements of this transient response at different positions over the primary visual cortex show a spatial pattern different from the one measured by electroencephalography. (C) 2008 Optical Society of America

OCIS codes: $030.6140,170.0170,170.5280,290.1990,290.1350,290.4210$.
\end{abstract}

Brain function relies on the constant supply of oxygen and glucose; in the healthy body, autoregulation provides a mechanism for adjustment of regional cerebral blood flow ( $\mathrm{rCBF}$ ) to very different physiological conditions. Under pathological conditions, such as after a stroke, autoregulation is restricted, and the continuous monitoring of rCBF is thus important for planning and assessment of therapy. Conventional methods to measure rCBF noninvasively (positronemission tomography, magnetic resonance, and x-ray tomography) rely on extrinsic, partially radioactive, markers and require large-scale installations that cannot be used in many clinically relevant situations, such as during surgery or in intensive care medicine. In the past few years, near-infrared diffusing-wave spectroscopy (DWS) has emerged as a powerful method for noninvasively quantifying blood flow in deep tissue based on the intrinsic contrast provided by the flow of erythrocytes [1-6]. DWS [also called diffuse correlation spectroscopy (DCS)] is the extension of quasi-elastic light scattering to the regime of strong multiple scattering $[7,8]$ and measures subwavelength scatterer motions deep within tissue by analyzing the temporal speckle fluctuations generated by a laser with a large coherence length. DWS autocorrelation functions measured over the human somato-motor cortex show a strongly accelerated decay within about $8 \mathrm{~s}$ after stimulation onset that reaches a saturation value for longer stimulation, which is consistent with the functionally enhanced $\mathrm{rCBF}$ measured by positron-emission tomography $[1,2,9,10]$. Steady-state visual stimulation by fullfield flickering leads to similar functional DWS signals measured over the primary visual cortex [3]. In contrast to motor stimulation, functional DWS signals from the primary visual cortex are considerably smaller, mainly owing to the larger distance of the primary visual cortex from the surface, and the temporal evolution of the rCBF to the steady state has not been accessible with neither DWS nor conventional methods. In this Letter we report on transient DWS signals during functional activation of the primary visual cortex using a fiber-based multispeckle detection setup. We observe an unexpected biphasic response of rCBF to full-field and checkerboard flickering suggesting a novel mechanism for adjustment of glucose supply to rapid stimuli.

Figures 1(a) and 1(b) schematically show the positioning of the optical probes over the visual cortex. Details of the instrument are described in [6]. Briefly, light from a diode laser (Toptica TA-100, $\lambda=802 \mathrm{~nm}$, coherence length $>100 \mathrm{~m}$ ) was guided to the scalp by an optical fiber. Diffusely transmitted light was collected by two fiber bundles $\left[R_{1}\right.$ and $R_{2}$ in Figs. 1(a) and $1(\mathrm{~b})]$ separated 1.5 and $3.0 \mathrm{~cm}$ from the source. The receiver fiber bundles $R_{1}$ and $R_{2}$ contain two and 28 few-mode fibers. The fibers in each bundle collect scattered light from a tissue surface area of about $1 \mathrm{~mm}^{2}$. The optical stimulation signal recorded by a few-mode fiber placed directly on the thin-film transistor (TFT) screen was used to precisely determine the onset of each stimulation block. Each receiver fiber was connected to an avalanche photodiode (Perkin-Elmer SPCM-AQ4C). Temporal autocorrelation functions $g^{(1)}(\tau)=\left\langle E(t) E^{*}(t+\tau)\right\rangle /\left\langle|E(t)|^{2}\right\rangle$ of the scattered electric field $E(t)$ at each fiber as well as average photon count rates $R=\left\langle|E(t)|^{2}\right\rangle$ from each of the avalanche photodiodes were simultaneously measured by using a 32-channel autocorrelator (correlator.com) at intervals of $26.2 \mathrm{~ms}$. Averaging the field autocorrelation functions from the statistically equivalent but uncorrelated speckles enhances the signal-to-noise ratio and allows one to detect small variations in the DWS signal associated with varying scatterer dynamics $[3,6]$. The decay of $g^{(1)}(\tau)$ was parametrized by its average decay time $\tau_{\mathrm{d}}$ [3]. At each time, the relative decay time $\tau_{\mathrm{s} / \mathrm{b}}$ and average photon 


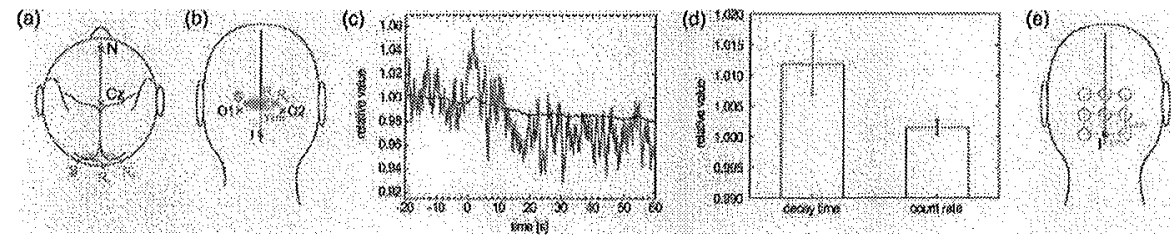

Fig. 1. (a) Top and (b) back view showing the positions of the source fiber $S$ and of the receiver fiber bundles $R_{1}$ and $R_{2}$ for the short- and the long-distance probes, respectively, over the primary visual cortex. $\mathrm{Cz}$, vertex; $\mathrm{N}$, nasion; $\mathrm{I}$, inion. (c) Stimulus-synchronized average of the relative decay time $\tau_{\mathrm{s} / \mathrm{b}}(\mathrm{red})$ and of the relative average photon count rate $R_{\mathrm{s} / \mathrm{b}}$ (blue) measured over the visual cortex with the long-distance sensor as a function of time after onset of visual stimulation $(t=0 \mathrm{~s})$, averaged over five subjects. Raw data were smoothed by a sliding average over $1 \mathrm{~s}$. (d) Group averages of the relative decay time $\tau_{\mathrm{s} / \mathrm{b}}$ and photon count rate $R_{\mathrm{s} / \mathrm{b}}$ averaged over the $8.2 \mathrm{~s}$ stimulation period. Optical probes were placed as shown in panel (a). Error bars represent the standard deviation for ten subjects. Student's $t$ test shows a significant functional increase of the DWS signal, while the count rate change is not significant. (e) Positioning of the DWS probes in the mapping experiment. The green circles mark the cortical areas probed with the different sensor positions.

count rate $R_{\mathrm{s} / \mathrm{b}}$ were determined by normalizing the decay time and average photon count rate values during stimulation by their respective averaged baseline values.

To detect the time evolution of the DWS signal during stimulation, we measured the stimulussynchronized average of the relative decay time $\tau_{\mathrm{s} / \mathrm{b}}$ with a block stimulation paradigm on five female subjects (ages 21-24) who were sitting comfortably in a chair during the entire experiment. A block measurement consisted of a $20 \mathrm{~s}$ baseline period followed by a $60 \mathrm{~s}$ stimulation period. During the stimulation period, the subjects were staring at a TFT screen $(43 \mathrm{~cm}$ diagonal at a distance of about $30 \mathrm{~cm}$ ) flickering at $7.5 \mathrm{~Hz}$; during the baseline period, the subjects fixated on a red cross in the center of the dark screen. The optical probes were located about $3.0 \mathrm{~cm}$ above the inion [see Figs. 1(a) and 1(b)]. Ten blocks of data were collected from each subject. The study protocol was approved by the university's Ethical Review Board.

During the $60 \mathrm{~s}$ stimulation period, the DWS signal shows a clear biphasic pattern [see Fig. 1(c)] within the first $2 \mathrm{~s}$ after the onset of the stimulus, the stimulus-synchronized $\tau_{\mathrm{s} / \mathrm{b}}$ increases rapidly up to about $6 \%$ beyond the baseline level, after which it decreases, crossing the baseline at about 6-10 s after the stimulus onset. At still longer times the DWS signal decreases further and, after about $15 \mathrm{~s}$, reaches a saturation value that is about $3 \%$ below the baseline level. This saturation value is in good agreement with our earlier observation of a reduction of $\tau_{\mathrm{s} / \mathrm{b}}$ by $3.0 \%-3.8 \%$ during $30 \mathrm{~s}$ full-field flickering stimulation [3]. At long times $t>20 \mathrm{~s}$ after onset of stimulation the photon count rate is reduced below its baseline value by about $1.6 \%$, indicating an increase in cortical blood volume that is in line with earlier nearinfrared spectroscopy (NIRS) studies [11]. At the short times $0 \mathrm{~s}<t \leqslant 2 \mathrm{~s}$, where we observe the large initial increase of $\tau_{\mathrm{s} / \mathrm{b}}$, the photon count rate $R$ increases slightly by about $0.5 \%$ beyond its value immediately before stimulation. The functional count rate signal is difficult to estimate owing to the presence of low-frequency fluctuations in the baseline that are of the order of $0.3 \%$ and are associated with the upright position of the subjects. Indeed, the change of the count rate is statistically not signifi- cant in the group of supine subjects (see below). The DWS signals and photon count rates from the shortdistance receiver $R_{1}$, which probes mainly the peripheral hemodynamics in the scalp and in the skull, show similar behavior as the data from the longdistance receiver, however, with strongly reduced amplitude (data not shown), suggesting that the short-distance receiver is partially sensitive to the hemodynamics in the cortex as well.

In the second experiment we focused on the initial response of the DWS signal during short stimulation blocks. During the 8.2 s stimulation blocks the subject stared at an $8 \times 6$ checkerboard flickering at $7.5 \mathrm{~Hz}$. The stimulation blocks were separated by baseline intervals of 8-12 s, which randomly varied to exclude synchronization of peripheral hemodynamics with the stimulus. The ten subjects that took part in the experiment (five male and five female, ages 19-26) were in supine position during the entire experiment. For each subject, 90 blocks of data were recorded.

Figure $1(d)$ shows the group average of the relative DWS signal $\tau_{\mathrm{s} / \mathrm{b}}$ and count rate $R_{\mathrm{s} / \mathrm{b}}$ recorded by the long-distance receiver and averaged over the $8.2 \mathrm{~s}$ stimulation period. The DWS signal shows a statistically significant increase by $(1.2 \pm 0.5) \%$ ( $t$ test: $t(9)$ $=2.39, p=0.04<0.05$ ). This result is consistent with the time-resolved data shown above, where the DWS decay time within the first $8 \mathrm{~s}$ after stimulation onset exceeds the baseline value. In contrast, the count rate increases by only $(0.15 \pm 0.12) \%$; this change is statistically not significant ( $t$ test: $t(9)=1.33, p$ $=0.21>0.05$ ). Similar to the observation in the timeresolved experiment, the DWS signal and the photon count rate recorded by the short-distance receiver parallel the behavior of the long-distance receiver data, albeit with considerably smaller amplitude.

To determine whether the unexpected increase of $\tau_{\mathrm{s} / \mathrm{b}}$ observed shortly after stimulation onset is due to a particular placement of the optical probe, we performed a mapping experiment on a subject in supine position (female, age 26) selected from the second subject group on the basis of the high photon count rate available. Optical probes with $3 \mathrm{~cm}$ sourcereceiver distance were placed at eight positions over the visual cortex successively, covering an area of $4 \mathrm{~cm} \times 4 \mathrm{~cm}$ directly above the inion [see Fig. 1(e)]. 


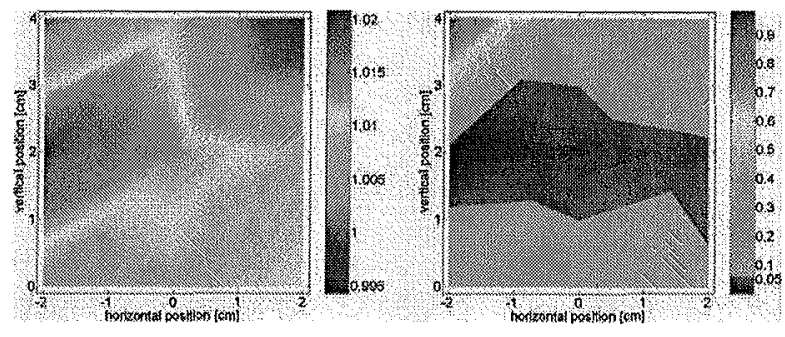

Fig. 2. Maps of the relative decay time $\tau_{\mathrm{s} / \mathrm{b}}$ (left) and of the corresponding $p$-value (right) during $8.2 \mathrm{~s}$ checkerboard stimulation over the visual cortex. The position $(0,0)$ corresponds to the inion. In the $p$-value map, regions of significant changes of $\tau_{\mathrm{s} b \mathrm{~b}}$ (i.e., with $p \leqslant 0.05$ ) are marked in blue. Maps are generated by piecewise bilinear interpolation based on the measured values at the eight sensor positions.

The stimulation protocol was identical with that of the previous experiment.

Figure 2 shows the maps for the DWS signal and the corresponding $p$ value over the visual cortex for $\tau_{\mathrm{s} / \mathrm{b}}$ averaged over the $8.2 \mathrm{~s}$ stimulation period. The $\tau_{\mathrm{s} / \mathrm{b}}$ map shows an increase by $1.0 \%-2.0 \%$ over a large area of the visual cortex, in particular in the left hemisphere, where the increase of $\tau_{\mathrm{s} / \mathrm{b}}$ is as much as $2.0 \%$. The $p$-value map shows that the most significant increase of $\tau_{\mathrm{s} / \mathrm{b}}$ occurs in a striplike region located $2 \mathrm{~cm}$ above the inion with a width of about $1 \mathrm{~cm}$. The observation that this laterally asymmetric pattern is clearly distinct from the symmetric activation pattern measured by electroencephalography for the same stimulation protocol (data not shown) indicates that the transient DWS signal is hemodynamic in origin.

The weak increase of the relative photon count rate $R_{\mathrm{s} / \mathrm{b}}$ by at most $0.5 \%$ for times $0 \mathrm{~s}<t \leqslant 2 \mathrm{~s}$ suggests a transient reduction of cortical blood volume. We estimate that the transient increase in $\tau_{\mathrm{s} / \mathrm{b}}$ expected for a reduction of $\mu_{\mathrm{s}}^{\prime}$ owing to reduced erythrocyte concentration in the tissue is at most $0.5 \%$ [12], which is by more than a decade below the DWS signal change of $6 \%$ observed at short times. If, on the other hand, we assume Grubb's scaling between changes in relative blood flow and relative blood volume to hold [12], we expect changes of the transient DWS signal of at most $1.5 \%$, which is about four times smaller than the observed DWS signal change. The large discrepancy between changes of blood volume and of blood flow for short stimuli indicates deviations from classical cerebrovascular autoregulation. We speculate that the large reduction of $\mathrm{rCBF}$ observed here provides a physiological mechanism for increasing the efficiency of glucose uptake into the cortical tissue shortly after onset of activation.

We thank M. Wolf, E. Keller and B. Rockstroh for helpful discussions. This work is funded by the Deutsche Forschungsgemeinschaft (DFG) and the Center for Applied Photonics (CAP) Konstanz.

\section{References}

1. T. Durduran, G. Yu, M. G. Burnett, J. A. Detre, J. H. Greenberg, J. Wang, C. Zhou, and A. G. Yodh, Opt. Lett. 29, 1766 (2004).

2. J. Li, G. Dietsche, D. Iftime, S. E. Skipetrov, G. Maret, T. Elbert, B. Rockstroh, and T. Gisler, J. Biomed. Opt. 10, 044002 (2005).

3. F. Jaillon, J. Li, G. Dietsche, T. Elbert, and T. Gisler, Opt. Express 15, 6643 (2007).

4. U. Sunar, H. Quon, T. Durduran, J. Zhang, J. Du, C. Zhou, G. Yu, R. Choe, A. Kilger, R. Lustig, L. Loevner, S. Nioka, B. Chance, and A. G. Yodh, J. Biomed. Opt. 11, 064021 (2006).

5. G. Yu, T. F. Floyd, T. Durduran, C. Zhou, J. Wang, J. A. Detre, and A. G. Yodh, Opt. Express 15, 1064 (2007).

6. G. Dietsche, M. Ninck, C. Ortolf, J. Li, F. Jaillon, and T. Gisler, Appl. Opt. 46, 8506 (2007)

7. G. Maret and P. E. Wolf, Z. Phys. B: Condens. Matter 65, 409 (1987).

8. D. J. Pine, D. A. Weitz, P. M. Chaikin, and E. Herbolzheimer, Phys. Rev. Lett. 60, 1134 (1988).

9. P. E. Roland, B. Larsen, N. A. Lassen, and E. Skinhøj, J. Neurophysiol. 43, 118 (1980).

10. R. J. Seitz and P. E. Roland, Eur. J. Neurosci. 4, 154 (1992).

11. M. Wolf, U. Wolf, V. Toronov, A. Michalos, L. A. Paunescu, J. H. Choi, and E. Gratton, Neuroimage 16, 704 (2002).

12. M. Jones, J. Berwick, D. Johnston, and J. Mayhew, Neuroimage 13, 1002 (2001). 